\title{
Pollution prevention with chemical process simulators: the generalized waste reduction (WAR) algorithm — full version
}

\author{
Heriberto Cabezas *, Jane C. Bare, Subir K. Mallick ${ }^{1}$ \\ U.S. Environmental Protection Agency, National Risk Management Research Laboratory, Sustainable Technology Division, \\ Systems Analysis Branch, 26 West Martin Luther King Drive, Cincinnati, OH 45268, USA
}

Received 11 February 1998; received in revised form 3 July 1998

\begin{abstract}
A general theory for the flow and the generation of potential environmental impact through a chemical process has been developed. The theory defines six potential environmental impact indexes that characterize the generation of potential impact within a process, and the output of potential impact from a process. The indexes are used to quantify pollution reduction and to develop pollution reducing changes to process flow sheets using process simulators. The potential environmental impacts are calculated from stream mass flow rates, stream composition, and a relative potential environmental impact score for each chemical present. The chemical impact scores include a comprehensive set of nine effects ranging from ozone depletion potential to human toxicity and ecotoxicity. The resulting waste reduction methodology or WAR algorithm is illustrated with two case studies using the chemical process simulator Chemcad III (Use does not imply USEPA endorsement or approval of Chemcad III). (C) 1999 Elsevier Science Ltd. All rights reserved.
\end{abstract}

\section{Introduction}

There is currently a great deal of interest in the development of methods that can be used to prevent or at least minimize the generation of pollution; and there are numerous efforts underway in this area (Lederman \& Weaver, 1991; El-Halwagi, El-Halwagi \& Manousiouthakis, 1992; Fonyo, Kurum \& Rippen, 1994; Mallick, Cabezas, Bare and Sikdar, 1996; Manousiouthakis and Allen, 1995; Rossiter, 1995). This interest stems from the belief that pollution prevention is likely to lead to the creation of technologies that have a much more benign impact on human health and the environment. Because this technology is inherently less polluting, it is likely to be more robust and economical than simply adding pollution control devices to conventional designs. In chemical manufacturing, these pollution prevention methods take the form of an effort to design process plants that generate as little pollution as

* Corresponding author. Fax: + 1-513-5697111.

E-mail address: cabezas.herberto@epamail.epa.gov (H. Cabezas)

${ }^{1}$ Post Doctoral Research Fellow, Oak Ridge Institute for Science and Education. Present address: Simulation Sciences Inc., 601 South Valencia Avenue, Brea, CA 92621, USA. possible. Since chemical process simulators are widely used in the design and operation of chemical manufacturing plants, the development of a pollution prevention methodology for chemical process simulators is likely to have a significant impact on the pollution generated by the chemical industry. At the National Risk Management Research Laboratory, research efforts are underway to develop a methodology for commercial chemical process simulators. The research effort is called the waste reduction or WAR algorithm after Hilaly and Sikdar (1994) who performed some of the early work in this area.

This paper presents a generalization of the WAR algorithm, discusses the methodology for evaluating potential environmental impacts, and illustrates the use of the method in the design or modification of chemical processes with two case studies.

\section{Potential environmental impact theory}

Potential environmental impact is the unrealized effect or impact that the emission of mass and energy would have on the environment on average. It is, 
therefore, essentially a probability function for the realization of a potential effect. Thus, the potential environmental impacts of chemical manufacturing processes are generally caused by the energy and material that the process takes from or emits to the environment. Potential environmental impact is a conceptual quantity that can not be directly measured, i.e. there are no potential environmental impact meters. However, one can calculate potential environmental impact from related measurable quantities using functional relations between the two. This situation is common in science and engineering. For example, the energy of a fluid can not be directly measured, but it can be calculated from temperature and pressure by the use of heat capacities and equations of state. Exactly how to perform a calculation for potential environmental impacts will be discussed later in this paper.

\subsection{Conservation equation}

Traditionally, chemical process design has been based on the creative application of mass and energy balances along with thermodynamics, chemical reaction engineering, and engineering economics. Our methodology proposes to add a conservation relation over potential environmental impact to the aforementioned two balance equations. The conservation equation for impacts is based on an accounting of the flow of potential environmental impact in and out of the processes. This flow of impact is related to the mass and energy flows but it is not equivalent to them. The impact conservation equation is

$\frac{\mathrm{d} I_{\text {syst }}}{\mathrm{d} t}=\dot{I}_{\text {in }}-\dot{I}_{\text {out }}+I_{\text {gen }}$

where $I_{\text {syst }}$ is the potential environmental impact content inside a process, $\dot{I}_{\text {in }}$ is the input rate of impact, $\dot{I}_{\text {out }}$ is the output rate of impact, and $\dot{I}_{\text {gen }}$ is the rate at which impact is generated in the system by chemical reactions or other means. Note that processes can also consume potential environmental impact so that $\dot{I}_{\text {gen }}$ can, in fact, be negative. For steady state processes, the conservation equation reduces to,

$0=\dot{I}_{\text {in }}-I_{\text {out }}+\dot{I}_{\text {gen }}$

which implies that no potential environmental impact accumulates in the system. Also note that Eqs. (1) and (2) serve as definitions of the function $\dot{I}_{\text {gen }}$.

The significance of potential environmental impacts can be better understood by considering the following definitions. If one were to dump into the environment all of the mass and energy flows entering a process, the resulting impact on the environment would equal to $I_{\text {in }}$; if one were to also dump into the environment all of the mass and energy flows exiting a process the resulting impact on the environment would be equal to $I_{\text {out }}$.
However, due to chemical transformations and changes in state conditions (temperature and pressure), $I_{\text {in }}$ is never exactly equal to $I_{\text {out }}$, and consequently $I_{\text {gen }}$ is never exactly equal to zero for steady state processes.

\subsection{Chemical processes}

Application of either Eq. (1) or Eq. (2) to chemical manufacturing processes requires an expression that relates the conceptual potential environmental impact to measurable quantities. Potential environmental impacts are caused by energy and material inputs and outputs to or from the environment. But, as a first approach, this treatment is restricted to potential impacts due to material flows while neglecting any impacts due to energy. Effects due to energy flows can be incorporated into the analysis by extending the boundary over which the impact balance is done to include the energy generation process. Effects due to resource depletion are also neglected mainly because there is no effective methodology for measuring them. This is consistent with the focus of this work which is the chemical process plant rather than a global life-cycle type of analysis. The expression relating potential chemical environmental impacts to measurables is

$\dot{I}_{i}=\sum_{j} \dot{I}_{j}^{(i)}=\sum_{j} \dot{M}_{j}^{(i)} \sum_{k} x_{k j} \psi_{j}+\ldots$

where the sum over $j$ is taken over the streams of input $i$ or output $i$, the sum over $k$ is taken over all chemicals $k, \dot{I}_{i}$ is the rate of potential environmental impact either in $\left(i=\right.$ in) or out of the process $(i=$ out $), \dot{I}_{j}^{(i)}$ is the rate of potential environmental impact for stream $j$ which may be an input or an output, $\dot{M}_{j}^{(i)}$ is the mass flow rate of stream $j$ which may again be either an input or an output, $x_{k j}$ is the mass fraction of chemical $k$ in stream $j$, and $\psi_{j}$ is the overall potential environmental impact of chemical $j$. Note that Eq. (3) is a first order approximation that does not include the synergistic effects that can occur when multiple chemicals are present.

\subsection{Impact indexes}

For steady state processes one can use Eq. (2) to define two categories of indexes for the environmental impact of chemical manufacturing. The first category of indexes measures the generation of potential environmental impact within processes, and the second one measures the potential environmental impact emitted by processes. There are various indexes that can be defined within each category. However, only the six indexes, three from each category, that seem most useful for waste reduction will be treated here.

Following Hilaly and Sikdar (1994), all non-products are considered to be pollutants and the potential environmental impact of all products is set to zero, i.e. 
$\psi_{j}=0$ for all products $j$. These assumptions are consistent with the objective of this paper which is to present a methodology for waste reduction, i.e. the primary concern is reducing the impact and the amount of the non-products. The broader implications of Eq. (1), including other impact indexes for which $\psi_{j} \neq 0$ for products $j$ and further conjectures on the implications for sustainability, will the subject of future publications.The first index of the first category of indexes (impact generation) is obtained by solving Eq. (2) for $\dot{I}_{\text {gen }}^{N P}$ and adding the superscript $N P$ for non-products to give,

$\dot{I}_{\text {gen }}^{N P}=\dot{I}_{\text {out }}^{N P}-\dot{I}_{\text {in }}^{N P}$

where $\dot{I}_{\text {out }}^{N P}$ and $\dot{I}_{\text {in }}^{N P}$ are the potential environmental impacts due to non-products, i.e. pollutants in the outputs and inputs, respectively. Eq. (3) is used to give explicit expressions for $\dot{I}_{\text {out }}^{N P}$ and $\dot{I}_{\text {in }}^{N P}$ where all components, products and non-products, are included in the summation, but where $\psi_{j}=0$ for all products $j$ which effectively removes all products from the summation. The index, $\dot{I}_{\text {gen }}^{N P}$, measures the total rate at which the process generates potential environmental impact due to non-products $(N P) . \dot{I}_{\mathrm{gen}}^{N P}$ has units of potential environmental impact generated per time.

The second index, $\hat{I}_{\text {gen }}^{N P}$, of the first category is obtained by dividing Eq. (4) by the rate at which the process generates products to give a specific impact generation,

$\hat{I}_{\mathrm{gen}}^{N P}=\frac{\dot{I}_{\mathrm{gen}}^{N P}}{\sum_{p} \dot{P}_{p}}=\frac{\dot{I}_{\mathrm{out}}^{N P}-\dot{I}_{\mathrm{in}}^{N P}}{\sum_{p} \dot{P}_{p}}$

where the sum over $p$ is taken over all the products $p$, and $\dot{P}_{p}$ is the mass flowrate of product $p . \hat{I}_{\mathrm{gen}}^{N P}$ measures the potential impact created by all non-products in manufacturing a unit mass of all the products $p$. The index, $\widehat{I}_{\mathrm{gen}}^{N P}$, has units of potential environmental impact per mass of products.

The third index of the first category, $\hat{M}_{\mathrm{gen}}^{N P}$, is obtained from Eq. (5) by setting the potential environmental impact $\left(\psi_{j}\right)$ of all products to zero and that of all non-products to one. This has the effect of assigning the same potential environmental impact to all nonproducts. The index, $\hat{M}_{\mathrm{gen}}^{N P}$, is a measure of the mass inefficiency of the process, i.e. it gives the ratio of mass converted to an undesirable form to mass converted to a desirable form. The expression for $\hat{M}_{\mathrm{gen}}^{N P}$ is

$$
\hat{M}_{\text {gen }}^{N P}=\frac{\sum_{j} \dot{M}_{j}^{(\text {out })} \sum_{k} x_{k j}^{N P}-\sum_{j} \dot{M}_{j}^{(\text {in })} \sum_{k} x_{k j}^{N P}}{\sum_{p} \dot{P}_{p}}
$$

where the summation over $\dot{M}_{j}^{\text {(out) }}$ is taken only over output streams, the summation over $\dot{M}_{j}^{(\text {in) }}$ is taken only over input streams, and the summation over $x_{k j}^{N P}$ is taken only over all non-products $k$ in stream $j$. The units of $\hat{M}_{\text {gen }}^{N P}$ are mass of non-products per mass of products.

The first index of the second category of indexes (impact emission) $\dot{I}_{\text {out }}^{N P}$ is simply the total rate of potential environmental impact output due to non-products. $\dot{I}_{\text {out }}^{N P}$ is calculated from Eq. (3) subject to $\psi_{j}=0$ for all products $j$. The index, $\dot{I}_{\text {out }}^{N P}$, is a measure of the rate at which the process emits potential environmental impact, and it has units of potential environmental impact per time.

The second index of the second category, $\hat{I}_{\text {out }}^{N P}$, is obtained by dividing the rate of potential environmental impact output due to non-products by the output rate of products to give,

$\hat{I}_{\mathrm{out}}^{N P}=\frac{\dot{I}_{\mathrm{out}}^{N P}}{\sum_{p} \dot{P}_{p}}$

The index, $\hat{I}_{\text {out }}^{N P}$, has units of potential environmental impact per mass of products. This expression gives the pollution index $\Phi$ of Mallick et al. (1996) which measures the potential environmental impact emitted in manufacturing a unit mass of products.

The third index of the second category, $\hat{M}_{\text {out }}^{N P}$, is obtained from Eq. (7) by setting the potential environmental impact $\left(\psi_{j}\right)$ of all products to zero and that of all non-products to one. The resulting expression is,

$$
\hat{M}_{\text {out }}^{N P}=\frac{\sum_{j} \dot{M}_{j}^{(\text {out })} \sum_{k} x_{k j}^{N P}}{\sum_{p} \dot{P}_{p}}
$$

which is related to the pollution index $\phi_{n}$ of Hilaly and Sikdar (1994) by,

$\hat{M}_{\mathrm{out}}^{N P}=\sum_{n} \phi_{n}$

where the summation is taken over all products $n . \hat{M}_{\text {out }}^{N P}$ measures the amount of non-product or pollutant mass emitted in manufacturing a unit mass of products, and it has units of non-product mass per mass of products. It is also a mass inefficiency measure.

\subsection{Significance of impact indexes}

The first category of indexes, i.e. $\dot{I}_{\mathrm{gen}}^{N P}, \hat{I}_{\mathrm{gen}}^{N P}$, and $\hat{M}_{\mathrm{gen}}^{N P}$, characterize some aspects of the generation of potential environmental impact within a manufacturing process. They are most useful in addressing questions related to the internal environmental efficiency of the process plant, i.e. the ability of the plant to produce desired products while creating a minimum of new, undesired potential environmental impact. It is important to note that once new potential environmental impact is created, resources such as potentially costly remediation 
efforts will likely be required to prevent the potential impact from being realized. Obviously, the smaller the values of $\dot{I}_{\mathrm{gen}}^{N P}, \hat{I}_{\mathrm{gen}}^{N P}$, and $\hat{M}_{\mathrm{gen}}^{N P}$ the more environmentally efficient the process, and, all others factors such as economics being equal, the more desirable. $\dot{I}_{\text {gen }}^{N P}$ would be useful in comparing different designs on an absolute basis, while $\widehat{I}_{\text {gen }}^{N P}$ and $\hat{M}_{\text {gen }}^{N P}$ would be useful in comparing different designs independently of manufacturing plant size.

The second category of indexes, i.e. $\dot{I}_{\text {out }}^{N P}, \hat{I}_{\text {out }}^{N P}$, and $\hat{M}_{\text {out }}^{N P}$, characterize some aspects of the emission of potential environmental impact from a manufacturing process. Their principal use is in addressing questions related to the external environmental efficiency of the process plant, i.e. the ability of the plant to produce desired products while inflicting on the environment a minimum of undesired potential environmental impact. It is again obvious that the smaller the values of $\dot{I}_{\text {out }}^{N P}$, $\hat{I}_{\text {out }}^{N P}$, and $\hat{M}_{\text {out }}^{N P}$, the more environmentally efficient the process, and all other factors such as economics being equal, the more desirable it is. Since $\dot{I}_{\text {out }}^{N P}$ is a total rate of impact output, it could be useful in deciding whether a given plant is compatible with a particular site. For example, it would be unwise to locate a plant with a high $\dot{I}_{\text {out }}^{N P}$ in an ecologically sensitive area. $\dot{I}_{\text {out }}^{N P}$ could also be used in matching the size of a plant to the capacity of the surrounding environment to dissipate environmental impact. $\hat{I}_{\text {out }}^{N P}$, and $\hat{M}_{\text {out }}^{N P}$ are more useful in comparing the potential environmental impact of alternative processes independently of plant size.

\section{Chemical environmental impact}

Application of the methodology so far developed requires that the potential environmental impacts of chemicals be estimated. Further, it is required that a relative impact number $\psi_{j}$ be given for each chemical $j$ over a wide range of different chemical environmental impacts. This is, unfortunately, not a trivial matter because chemical impacts are measured on different relative scales that can not be simply added without some form of normalization.

\subsection{Chemical impact expression}

To apply the WAR methodology to chemical processes, the following expression for $\psi_{j}$ has been developed (Mallick et al. 1996),

$\psi_{j}=\sum_{l} \alpha_{l} \psi_{j, l}^{s}$

where the sum is taken over categories of potential chemical environmental impacts, e.g. ozone depletion potential, human health, etc. listed below under Section 3.2. $\alpha_{l}$ is a relative weighting factor for impact of type $l$ independent of chemical $j$, and $\psi_{j, l}^{s}$ is the specific potential environmental impact of chemical $j$ for an impact of type $l . \alpha_{l}$ has units of potential environmental impact per mass.

The relative weighting factor $\alpha_{l}$ allows Eq. (10) to be customized to specific or local conditions. The suggested procedure is to initially set all the $\alpha_{l}$ 's to same value of say one, and to allow users to vary individual $\alpha_{l}$ 's from 0 to 10 according to local needs and policies. Please note that for a relative comparison, the absolute value is not critical. For example, photochemical oxidation potential would be weighted more heavily than other impacts in an area that suffers from smog. There is an effort underway in our research group to develop more sophisticated methods of determining values for the $\alpha_{l}$ 's.

The values for the $\psi_{j, l}^{s}$ were obtained from the relative rankings or scores for chemicals by normalizing according to,

$\psi_{i, j}^{s}=\frac{(\text { Score })_{i, j}}{\left\langle(\text { Score })_{i}\right\rangle_{j}+2 \sigma_{j}}$

where (Score) $)_{i, j}$ is the relative score of chemical $i$ on some arbitrary scale within impact category $j$, $\left\langle(\text { Score })_{i}\right\rangle_{j}$ is the arithmetic average of the scores of all chemicals $i$ within impact category $j$, and $\sigma_{j}$ is the standard deviation of all the chemical scores in impact category $j$. The normalizing factor $\left\langle(\text { Score })_{i}\right\rangle_{j}+2 \sigma_{j}$ assures that about $75 \%$ of the normalized chemical score numbers $\psi_{j, l}^{s}$ will be between 0 and 1 irrespective of the statistical distribution of the initial scores as expected from Chebyshev's theorem (Lapin, 1975). If the chemical scores happen to follow a normal distribution, then the normalization range extends to $\approx 95 \%$ of the scores.

\subsection{Classification of impacts}

The classification of chemical environmental impacts and the values for the (Score) $)_{i, j}$ were adopted from the study of Heijungs, Guinée, Huppes, Lankreijer, Udo de Haes and Wegener Sleeswijk $(1992)^{2}$ and normalized according to Eq. (11) to obtain the $\psi_{j, l}^{s}$ 's. In the calculation of $\left\langle(\text { Score })_{i}\right\rangle_{j}$ and $\sigma_{j}$, the chemical scores for dioxin, chromium VI, and vinyl chloride were excluded. The reason is that the score numbers for these three chemicals were several orders of magnitude higher that those for all other chemicals, and including them in the normalization process would have made the normalization meaningless. Therefore, the normalized $\psi_{j, l}^{s}$ for these three chemicals would appear as outliers which they, in fact, are.

\footnotetext{
${ }^{2}$ Use by the authors does not imply endorsement or approval by the U.S. Environmental Protection Agency.
} 
There are nine different impact categories. These can be subdivided into four environmental physical potential effects (acidification, greenhouse enhancement, ozone depletion, and photochemical oxidant formation), three human toxicity effects (air, water, and soil), and two ecotoxicity effects (aquatic and terrestrial).

The normalized chemical scores used in the two case studies presented in the next sections are given in Table 1 below where $\mathrm{H}_{2}$ is hydrogen, MEK is methyl ethyl ketone, SBA is secondary butyl alcohol, $\mathrm{H}_{2} \mathrm{O}$ is water, $\mathrm{N}_{2}$ is molecular nitrogen, $\mathrm{Ar}$ is argon, $\mathrm{CH}_{4}$ is methane, and $\mathrm{NH}_{3}$ is ammonia. These acronyms will be used throughout the rest of the paper.

\section{Case study \# 1: MEK production}

To illustrate the use of the generalized WAR algorithm, a case study from the production of methyl ethyl ketone (MEK) from secondary butyl alcohol (SBA) is presented. The case study was adopted from the Chemcad III (Chemstations, 1997) chemical process simulator, and all the material and energy balances were performed using Chemcad III. However, any commercial process simulator could have been used. This case study presents a typical chemical engineering process for the production of a commodity chemical that involves several unit processes such as reactors, separators, mixers, dividers, and heat exchangers. It is, therefore, sufficiently complex to illustrate the WAR algorithm but still treatable within the space of this paper. Essentially, the chemical process consists of a SBA dehydrogenation reactor followed by a MEK purification train and associated equipment.

\subsection{Base flowsheet}

Fig. 1 shows the base process flow diagram for the production of MEK from SBA. SBA is fed to a hydrogen scrubber where the feed SBA scrubs residual MEK from the hydrogen stream. The SBA feed is then pumped up to reaction pressure and heated to reaction temperature with a heat exchanger and a heater. The heated SBA is fed to the reactor where the chemical transformation occurs. The reactor output stream is sent to a heat exchanger where it is partially condensed. The mixture of MEK, hydrogen, and unconverted SBA

Table 1

Normalized chemical impact scores

\begin{tabular}{llll}
\hline $\mathrm{H} 2$ & MEK & SBA & $\mathrm{H} 2 \mathrm{O}$ \\
\hline 0 & 0.42 & $4.1 \mathrm{E}-4$ & 0 \\
$\mathrm{~N} 2$ & $\mathrm{Ar}$ & $\mathrm{CH} 4$ & $\mathrm{NH} 3$ \\
0 & 0 & $7.4 \mathrm{E}-3$ & 0.93 \\
\hline
\end{tabular}

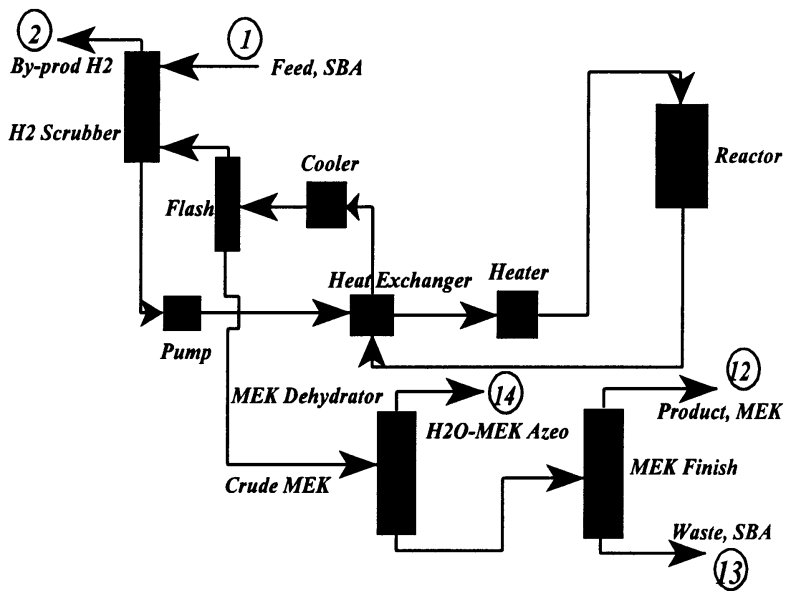

Fig. 1. Base process flow diagram for the production of methyl ethyl ketone from secondary butyl alcohol.

is cooled further and sent to a separator where the hydrogen is flashed off. The hydrogen is then scrubbed and the liquid phase fed to a MEK purification system. The mass flow rates and the state of the various input and output streams as calculated by Chemcad III are listed in Table 2 above.

\subsection{Modified flowsheet}

Examination of the base process indicates that waste stream 13 contains large amounts of unreacted SBA and small amounts of MEK. It is, therefore, logical to try to recover the SBA and MEK as the first step in a waste reduction strategy. Consequently, the process flow diagram was modified by the addition of a recycle from stream 13 to the feed, stream 1 . Two cases were studied with this modification, recycling 50 and $100 \%$ of stream 13 . Recycling $100 \%$ of stream 13, i.e. closing off stream 18, is appropriate for this illustration. But, it is clearly not practical because stream 18 is the only liquid waste stream in the modified process and the only means of purging the system of liquid impurities. Without this purge, impurities would build up inside the process causing it to eventually cease to function. Fig. 2 below shows the flow diagram for the modified process. The mass flow rates and the state of the various input and output streams for the modified process with $50 \%$ recycle are listed in Table 3

The process modification increased the amount of product by $\approx 73 \%$ while reducing the amount of waste SBA in stream 18 by $\approx 20 \%$.

It is important to note that an examination of Tables 2-4 will indicate that waste was generally reduced, and that environmental impact was probably also reduced. However, the information so far considered is not sufficient to allow a quantitative comparison of the overall waste and environmental impact reduction associated with each of the three cases studied here. For this 
Table 2

MEK production flow summary $(\mathrm{kg} / \mathrm{hr})$ input \& output: base process

\begin{tabular}{|c|c|c|c|c|c|}
\hline SBA & 3362 & 19 & 3 & 2670 & 1 \\
\hline MEK & 0 & 0 & 567 & 13 & 71 \\
\hline $\mathrm{H} 2$ & 0 & 18 & 0 & 0 & 0 \\
\hline
\end{tabular}

comparison one must calculate the impact indexes already described.

For the modified process with $100 \%$ recycle, the mass flow rates and the state of the various input and output streams are listed in Table 4 below. Note that increasing the recycle increased the amount of product by $269 \%$ while simultaneously reducing the amount of waste SBA in stream 13 to zero.

\subsection{Impact index calculations}

Six different impact indexes were calculated for the base case and the two modified processes each. The indexes were obtained using Eqs. (3)-(8), the flow rates from Tables 2-4, Eq. (10), and the normalized chemical impact scores of Table 1 . The relative weighting factors, $\alpha_{l}$, were all set to one for these calculations.

The first category indexes, i.e. the impact generation indexes, $\dot{I}_{\text {gen }}^{N P}, \hat{I}_{\text {gen }}^{N P}$, and $\hat{M}_{\text {gen }}^{N P}$ are shown on Fig. 3 below. It should be noted that $\hat{M}_{\text {gen }}^{N P}$ is a negative number since some of the input mass is always converted to product, and the products are not included in the summation of the outputs. The specific indexes, $\widehat{I}_{\text {gen }}^{N P}$, and $\hat{M}_{\text {gen }}^{N P}$ were multiplied by a factor of 100 so that they could be shown on the same scale as the rate index.

The second category indexes, i.e. the impact output indexes $\dot{I}_{\text {out }}^{N P}, \hat{I}_{\text {out }}^{N P}$, and $\hat{M}_{\text {out }}^{N P}$, are shown in Fig. 4. The specific index $\hat{I}_{\text {out }}^{N P}$ was multiplied by a factor of 1000 , and the specific index $\hat{M}_{\text {out }}^{N P}$ was multiplied by a factor of 10 so that they could both be shown on the same scale as $\dot{I}_{\text {out }}^{N P}$. The largest source of uncertainty in the calculation of the impact indexes is the environmental impact scores. These measurements are probably accurate to no more than one significant figure or an order of magnitude. It is, therefore, prudent to assume that impact index calculations are also accurate to no more than one significant figure. Two significant figures are used in Figs. 3 and 4 in order to help the readers to reproduce the calculations, if necessary.

\section{Discussion of case study \# 1}

The impact generation indexes of Fig. 3 show that: (1) the rate of impact generation by non-products de- creases by $13 \%$ for $50 \%$ recycle and by $20 \%$ for $100 \%$ recycle, (2) the impact generated by non-products $\mathrm{kg}^{-1}$ of product decreases by $48 \%$ for $50 \%$ recycle and by $77 \%$ for $100 \%$ recycle, and (3) the kilograms of nonproducts generated $\mathrm{kg}^{-1}$ of product remains nearly constant at -100 . The most significant index in this case is the impact generated $\mathrm{kg}^{-1}$ of product. The decrease of this index reflects the increase in the productivity of the plant, i.e. the increase in product flow rate.

The impact output indexes of Fig. 4 show that: (1) the rate of impact output from non-products decreases by $11 \%$ for $50 \%$ recycle and by $17 \%$ for $100 \%$ recycle, (2) the impact output from non-products $\mathrm{kg}^{-1}$ of product decreases by $48 \%$ for $50 \%$ recycle and by $78 \%$ for $100 \%$ recycle, and (3) the output of kilograms of non-product $\mathrm{kg}^{-1}$ of product decreases by $60 \%$ for $50 \%$ recycle and by $88 \%$ for $100 \%$ recycle. It is worth noting that the output of impact and waste decreased as measured by all the indexes. The most significant index in this case is the impact output $\mathrm{kg}^{-1}$ of product. The decrease in this index is consistent with the decrease in the impact generation per mass of product index discussed in the paragraph above. This decrease is also a reflection of the increased productivity of the plant.

The decreases in the indexes are sufficiently large such that they represent significant reductions in pollution. The consistent decrease in the impact generation per mass of product $(48-77 \%)$ and the impact output

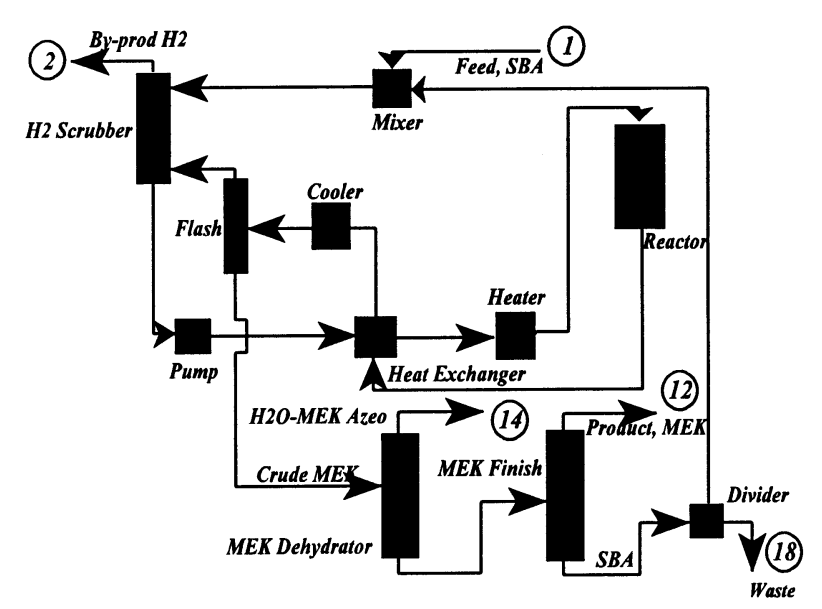

Fig. 2. Modified process flow diagram for the production of methyl ethyl ketone from secondary butyl alcohol. 
MEK production flow summary $(\mathrm{kg} / \mathrm{hr})$ input \& output: modified process $(50 \%$ recycle)

\begin{tabular}{lrrrrr}
\hline Stream (state) & $\# 1(\mathrm{~L})$ & $\# 2(\mathrm{G})$ & $\# 12(\mathrm{~L})$ & $\# 14(\mathrm{G})$ & \# $18(\mathrm{~L})$ \\
\hline SBA & 3362 & 168 & 5 & 1 & 2124 \\
MEK & 0 & 1 & 980 & 6 & 10 \\
H2O & 8 & 2 & 0 & 0 & 0 \\
H2 & 0 & 29 & 0 & 0 \\
\hline
\end{tabular}

per mass of product $(48-78 \%)$, simply means that the modified plant can meet the needs of a much larger market without increasing its generation or its output of potential environmental impact. It also means that a modified plant that is $48-77 \%$ smaller than the base case can meet the needs of the same market that the base plant was designed for.

\section{Case study \# 2: ammonia production}

To further illustrate the use of the generalized WAR Algorithm, a second case study from the production of ammonia from synthesis gas is presented. The case study was also adopted from the Chemcad III (Chemstations, 1997) chemical process simulator, and all the material and energy balances were performed using Chemcad III. However, any commercial process simulator could again have been used. Just as case study \#1, this case study also presents a typical chemical engineering process that involves several unit processes such as reactors, separators, mixers, dividers, and heat exchangers. It is also sufficiently complex to illustrate the WAR algorithm but still treatable within the space of this paper.

\subsection{Base flowsheet}

Fig. 5 shows the base process flow diagram for the process. Essentially, the overall process is based on the reaction of nitrogen and hydrogen to produce ammonia.

The mixture of hydrogen and nitrogen is compressed and cooled and feed to a series of three reactors through a flash drum. Several reactors are normally used to maximize the conversion of feed to products which for this process is difficult to do. This flash drum also serves to separate the ammonia product from the unreacted gases which are feedback into the system. The ammonia is recovered as an anhydrous liquid under modest pressure. Themass flow rates and the state of the input and output streams as calculated by Chemcad III are all listed in Table 5.

\subsection{Modified flowsheet}

Examination of the base process indicates that waste stream 17 contains ammonia and some unreacted feed.
It is, therefore, logical to try to recover the ammonia and the unreacted feed as an obvious first step in a waste reduction strategy. Consequently, the process flow diagram was modified in two ways. First, the purge ratio was reduced five fold from 0.1 to 0.02 , i.e. the flow of stream 17 was reduced 5 -fold. Second, in addition to reducing the purge, a flash drum was added with stream 17 as the feed to recover some of the ammonia.

Fig. 5 shows the configuration of the flow diagram for the reduced purge modified process which is identical to that of the base process. The mass flow rates and the state of the input and output streams for the reduced purge modified process are listed in Table 6 . The process modification increased the amount of product by $\approx 25 \%$ while reducing the amount of waste ammonia in stream 17 by $\approx 77 \%$. It is worth noting, however, that there was an small increase in the impurities present in the product stream. This changed from about $2 \%$ impurities in the base case to $3 \%$ for the reduced purge modified case.

Fig. 6 shows the flow diagram for the modified process with reduced purge and addition of a flash drum with stream 17 as the feed. Under this configuration, stream 25 is used to purge impurities from the system. Without this purge, impurities would again build up inside the process, and it would eventually cease to function. Stream 24 which consists of essentially pure ammonia is mixed with stream 19 to form a new product stream, stream 26.

The mass flow rates and the state of the various input and output streams are listed in Table 7. Note that adding the flash drum in addition to reducing the purge 5 -fold increased the amount of product by $26 \%$ as compared to the base case. This is very close to the $25 \%$ increase in product that was obtained by simply reducing the purge. However, the amount of waste ammonia in stream 25 was reduced by $91 \%$, and the amount of total waste in stream 25 was reduced by $78 \%$, both

Table 4

MEK production flow summary $(\mathrm{kg} / \mathrm{hr})$ input \& output: modified process $(100 \%$ recycle)

\begin{tabular}{lrrrr}
\hline Stream (state) & \# $1(\mathrm{~L})$ & \# $2(\mathrm{G})$ & \# $12(\mathrm{~L})$ & \# $14(\mathrm{G})$ \\
\hline SBA & 3362 & 1117 & 11 & 1 \\
MEK & 0 & 11 & 2094 & 59 \\
H2O & 8 & 3 & 0 & 5 \\
H2 & 0 & 60 & 0 & 0
\end{tabular}




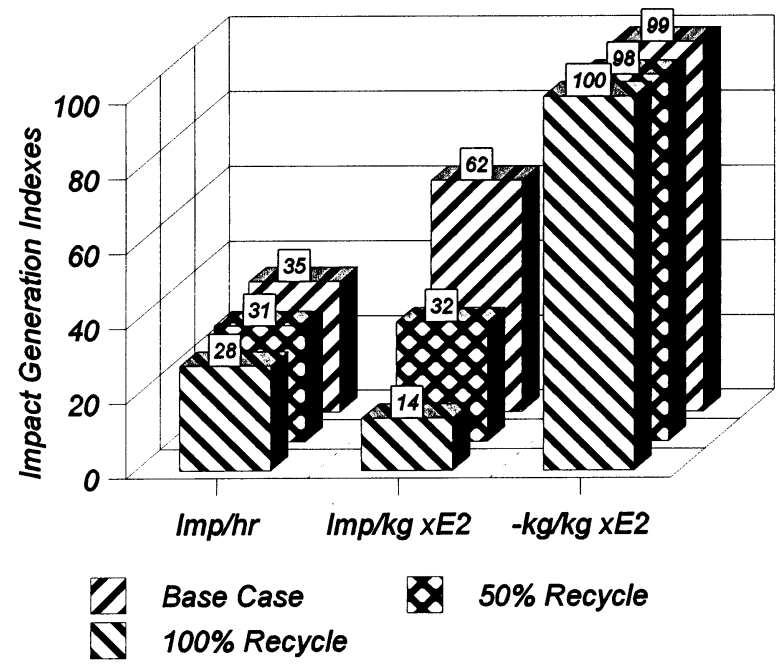

Fig. 3. Impact generation indices for the production of methyl ethyl ketone from secondary butyl alcohol $\dot{I}_{\text {out }}^{N P}$ in impact units of pollutants $\mathrm{h}^{-1}, \hat{I}_{\text {out }}^{N P}$ in impact units of pollutants $\mathrm{kg}^{-1}$ of products, and $\hat{M}_{\text {out }}^{N P}$ in $\mathrm{kg}$ of pollutants $\mathrm{kg}^{-1}$ of product.

compared to the base case. As compared to the reduced purge process, the addition of the flash drum increased the amount of product by a meager $1 \%$, but it reduced the amount of waste ammonia by $61 \%$ and total amount of waste by $18 \%$, both in stream 25 . Therefore, the principal effect of adding the flash drum was the reduction of waste.

An examination of Tables 5-7 will again show that waste was generally reduced, that the amount of product made was increased, and that environmental impact of the process was probably also reduced. However, one finds that it is not sufficient to allow a quantitative comparison of the overall waste and environmental impact associated with each of the three

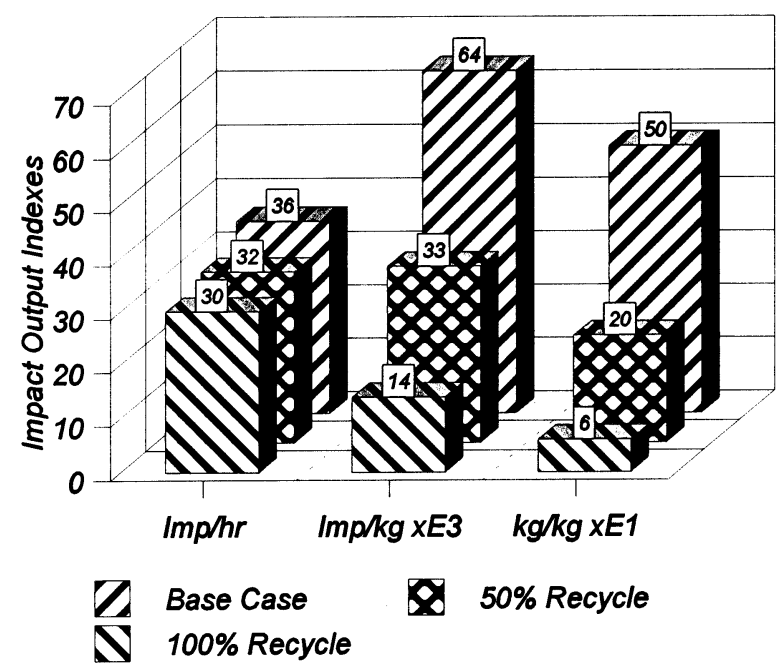

Fig. 4. Impact output indexes for the production of methyl ethyl ketone from secondary butyl alcohol: $\dot{I}_{\text {out }}^{\mathrm{NP}}$ in impact units of $\mathrm{kg} \mathrm{h}^{-1}$, $\hat{I}_{\text {out }}^{\mathrm{NP}}$ in impact units of pollutants $\mathrm{kg}^{-1}$ of products, and $\hat{M}_{\text {out }}^{\mathrm{NP}}$ in $\mathrm{kg}$ of pollutants $\mathrm{kg}^{-1}$ of products.

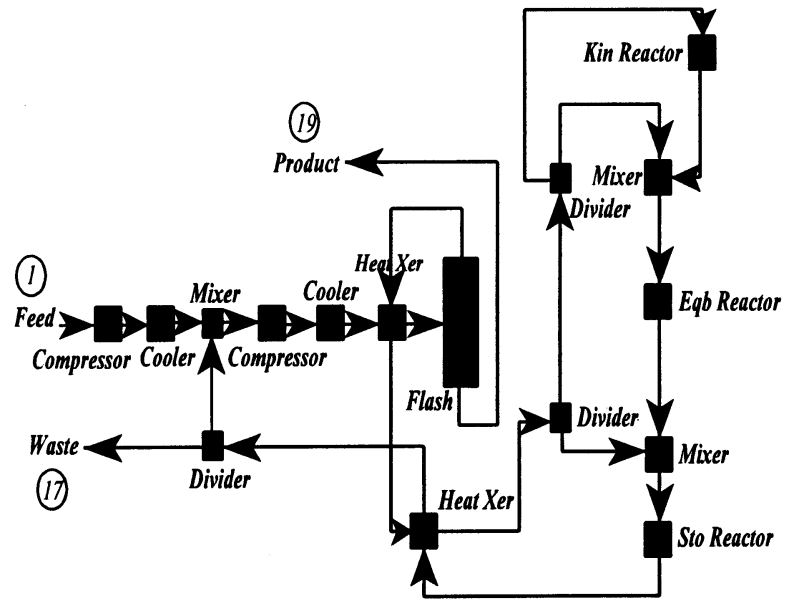

Fig. 5. Base process flow diagram for the production of ammonia from synthesis gas.

cases. In a simple example such as this one, the task is confusing, but for complex chemical processes it can become impossible. For this comparison one must calculate the impact indexes.

\subsection{Impact index calculations}

Again, six different impact indexes were calculated for the base and the two modified processes. The indexes were also obtained using Eqs. (3)-(8), the flow rates from Tables 5-7, Eq. (10), and the normalized chemical impact scores of Table 1 . The relative weighting factors were all set to one for these calculations.

The first category indexes, i.e. the impact generation indexes, $\dot{I}_{\text {gen }}^{N P}, \hat{I}_{\text {gen }}^{N P}$, and $\hat{M}_{\text {gen }}^{N P}$, are shown on Fig. 7. It should be noted that $\hat{M}_{\text {gen }}^{N P}$ is a negative number since some of the input mass is always converted to product, and the products are not included in the summation of the outputs. The specific index, $\hat{I}_{\text {gen }}^{N P}$, was multiplied by a factor of 10000 , and the index, $\hat{M}_{\text {gen }}^{N P}$, was multiplied by a factor of 1000 so that they could both be shown on the same scale as the rate index.

The second category indexes, i.e. the impact output indexes, $\dot{I}_{\text {out }}^{N P}, \hat{I}_{\text {out }}^{N P}$, and $\hat{M}_{\text {out }}^{N P}$, are shown in Fig. 8. The specific index $\hat{I}_{\text {out }}^{N P}$ and the specific index $\hat{M}_{\text {out }}^{N P}$ were both multiplied by a factor of 10000 so that they could be shown on the same scale as $\dot{I}_{\text {out }}^{N P}$.

Table 5

Ammonia production flow summary $(\mathrm{kg} / \mathrm{hr})$ input \& output: base process (purge ratio $=0.1$ )

\begin{tabular}{lrrr}
\hline Stream (state) & $\# 1(\mathrm{G})$ & $\# 17(\mathrm{G})$ & $\# 19(\mathrm{~L})$ \\
\hline $\mathrm{N} 2$ & 33334 & 5060 & 187 \\
$\mathrm{Ar}$ & 603 & 428 & 176 \\
$\mathrm{H} 2$ & 7196 & 1120 & 13 \\
$\mathrm{CH} 4$ & 805 & 700 & 112 \\
NH3 & 0 & 3696 & 30453 \\
\hline
\end{tabular}


Table 6

Ammonia production flow summary $(\mathrm{kg} / \mathrm{hr})$ input \& output: modified process (purge ratio $=0.02$ )

\begin{tabular}{lrrr}
\hline Stream (state) & $\# 1(\mathrm{G})$ & $\# 17(\mathrm{G})$ & $\# 19(\mathrm{~L})$ \\
\hline $\mathrm{N} 2$ & 33334 & 1162 & 217 \\
$\mathrm{Ar}$ & 603 & 199 & 404 \\
$\mathrm{H} 2$ & 7196 & 281 & 16 \\
$\mathrm{CH} 4$ & 805 & 446 & 351 \\
NH3 & 0 & 856 & 38001 \\
\hline
\end{tabular}

The largest source of uncertainty in the calculation of the impact indexes are again the environmental impact scores. These measurements are probably accurate to no more than one significant figure or an order of magnitude, and it is, therefore, prudent to assume that impact index calculations are also accurate to no more than one significant figure. Two significant figures are presented in Figs. 7 and 8 in order to allow readers to reproduce the calculations if necessary.

\section{Discussion of case study \#2}

The impact generation indexes of Fig. 7 show that: (1) the rate of impact generation by non-products decreases by $77 \%$ when the purge ratio is decreased from 0.1 to 0.02 and by $91 \%$ when the purge ratio is decreased as above and a flash drum is added to recover waste ammonia; (2) the impact generated by non-products $\mathrm{kg}^{-1}$ of product decreases by $81 \%$ when the purge ratio is reduced from 0.1 to 0.05 and by $93 \%$ when the purge ratio is decreased as above and a flash drum is added; and (3) the kilograms of non-products generated $\mathrm{kg}^{-1}$ of product remained nearly constant at -1 for all cases. The most significant index in this case is the impact generated $\mathrm{kg}^{-1}$ of product. The decrease of this index reflects primarily the recovery of the waste

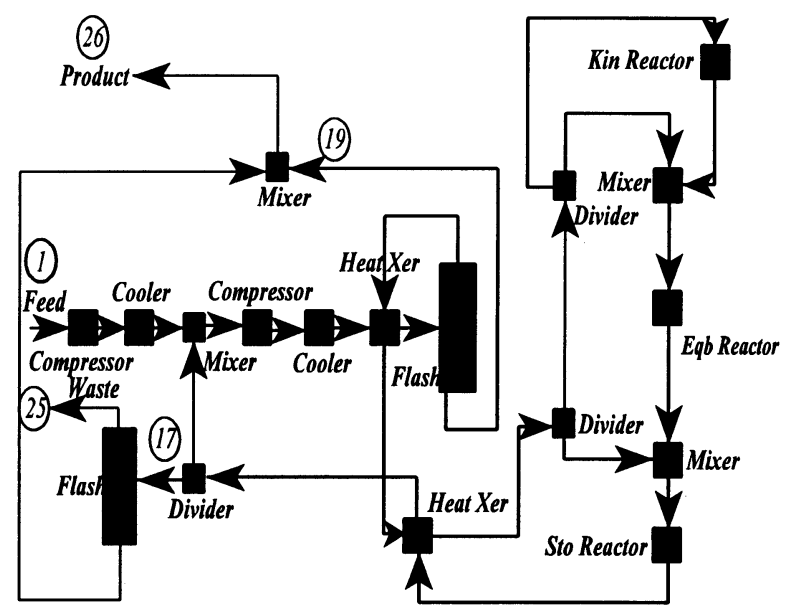

Fig. 6. Modified process flow diagram for the production of ammonia from synthesis gas with reduced purge ratio and added flush drum.
Table 7

Ammonia production flow summary $(\mathrm{kg} / \mathrm{hr})$ input \& output: modified process (purge ratio $=0.02 \&$ flash drum)

\begin{tabular}{lrrr}
\hline Stream (state) & $\# 1(\mathrm{G})$ & $\# 25(\mathrm{G})$ & $\# 26(\mathrm{~L})$ \\
\hline N2 & 33334 & 1162 & 217 \\
Ar & 603 & 199 & 404 \\
H2 & 7196 & 281 & 16 \\
CH4 & 805 & 447 & 351 \\
NH3 & 0 & 335 & 38521 \\
\hline
\end{tabular}

product and to a smaller extent the increase in the productivity of the plant, i.e. the increase in product flow rate.

The impact output indexes of Fig. 8 show that: (1) the rate of impact output from non-products decreases by $76 \%$ when the purge ratio is decreased from 0.1 to 0.02 and by $91 \%$ when the purge ratio is decreased as above and a flash drum is added to recover waste ammonia; (2) the impact output from non-products $\mathrm{kg}^{-1}$ of product decreases by $81 \%$ when the purge ratio is reduced from 0.1 to 0.05 and by $93 \%$ when the purge ratio is decreased as above and a flash drum is added; and (3) the output of kilograms of non-product $\mathrm{kg}^{-1}$ of product decreases by $73 \%$ when the purge ratio is reduced from 0.1 to 0.05 and by $76 \%$ when the purge ratio is decreased as above and a flash drum is added. It is again worth noting that the output of impact and waste decreased as measured by all the indexes. The most significant index in this case is the impact output $\mathrm{kg}^{-1}$ of product. The decrease in this index was the same as that for the equivalent generation index. This is a reflection of the change in the index being primarily driven by the recovery of waste product rather than increased productivity.

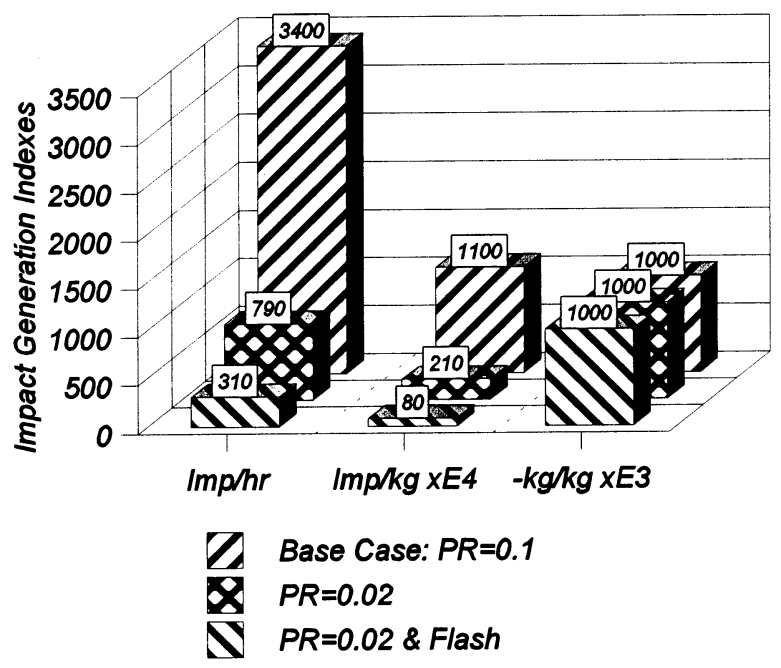

Fig. 7. Impact generation indexes for the production of ammonia from synthesis gas: $I_{\text {gen }}^{N P}$ in impact units $\mathrm{h}^{-1}, \hat{I}_{\text {gen }}^{N P}$ in impact units $\mathrm{kg}^{-1}$ of product, and $-\hat{M}_{\mathrm{gen}}^{N P}$ in $\mathrm{kg}$ of pollutants $\mathrm{kg}^{-1}$ of product. $\mathrm{PR}$ is the purge ratio. 


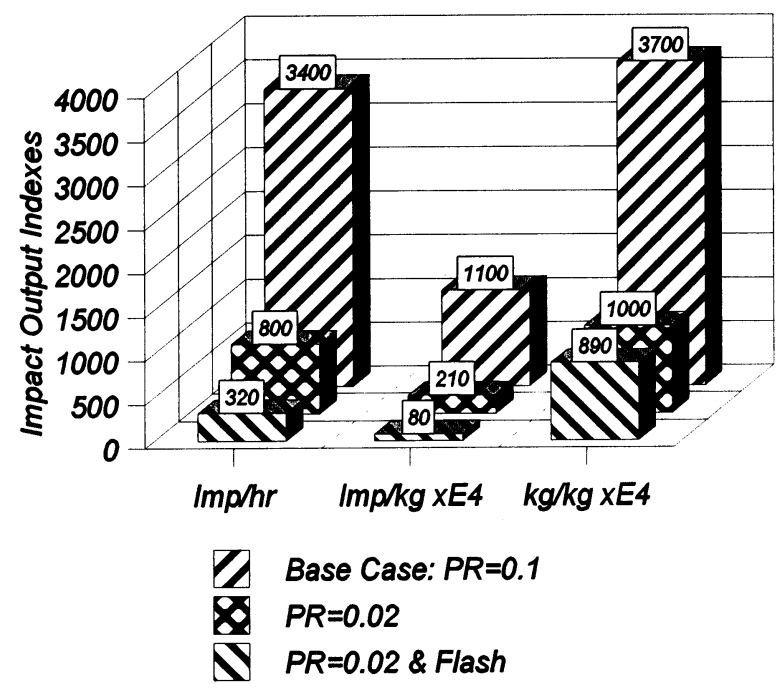

Fig. 8. Impact output indexes for the production of ammonia from synthesis gas: $\dot{I}_{\text {out }}^{N P}$ in impact units of pollutants $\mathrm{h}^{-1}, \hat{I}_{\text {out }}^{N P}$ in impact units of pollutants $\mathrm{kg}^{-1}$ of products, and $\hat{M}_{\text {out }}^{N P}$ in $\mathrm{kg}$ of pollutants $\mathrm{kg}^{-1}$ of products. PR is the purge ratio.

The decreases in the indexes are again sufficiently large that they represent significant reductions in pollution. The consistent decrease in the impact generation per mass of product and impact output per mass of product $(81 \%$ to $93 \%)$ implies that the modified plant can meet the needs of a moderately larger market with much less impact on the environment. The decrease in the rate of impact generation and impact output ( $76 \%$ to $91 \%$ ) means that the modified process has an environmental impact that is roughly one tenth that of the base plant. Lastly, the decrease in the output of waste mass per mass of product indicates that the modified plant will lose less valuable material in its operation.

\section{Future work}

In addition to the topics already mentioned, there are two other issues that need to be further mentioned in relation to the WAR algorithm: engineering economics and computer aided process design. However, both of these are beyond the scope of this paper which is to present the basic generalized waste reduction or WAR algorithm and to illustrate its use. Engineering economics and computer aided process design are the subject of present and future research, and they are included here only for completeness and to aid interested readers applying the method and furthering the work.

\subsection{Engineering economics}

Whenever a process is modified to reduce waste, there is a consequent change in the economics associated with it. Economic changes are generally due to: (1) the addition or removal of capital equipment; (2) an increase or decrease in energy consumption; (3) a change in the rate of consumption of feed material; and (4) a change in the rate of product generation. When a process is modified, all of the above are frequently affected. There are well established methods for estimating the economics of chemical processes either manually (Peters \& Timmerhous, 1968; Richardson Engineering Services, 1997) or with the aid of a computer (Aspen Technology, 1997; ICARUS Corporation, 1997).

Modification of a chemical process using the WAR algorithm needs to be done along with an evaluation of the economic consequences of any proposed process modifications, i.e. one needs to simultaneously compare both the potential environmental impact and the cost of alternate process flowsheets. The reason is that the ultimate objective is always that of developing cost effective reductions in pollution. Unfortunately, there are no consensus criteria for cost effective waste reduction. Although one possibility, consistent with traditional process design procedures, is to require that the sum of the capital and operating costs should not increase with proposed process modifications from the base configuration. This can be expressed as,

$\left[C_{o}+C_{c}\right]_{\text {Base }} \geq\left[C_{o}+C_{c}\right]_{\text {Modified }}$

where $C_{o}$ is the operating cost and $C_{c}$ is the capital cost that can be estimated by one of the aforementioned methods or some another proprietary method. Eq. (12) can then be used jointly with Eqs. (4)-(8) to evaluate alternative process flowsheets.

\subsection{Computer aided process design}

While it is often possible to devise pollution reducing modifications from an inspection of the process flowsheet, there are many situations where a more systematic approach such as computer aided process design and optimization may be required. This is particularly important with very complex processes that are difficult to analyze by inspection. The WAR algorithm can be used in computer aided process design and optimization. This can be done by employing the indexes of Eqs. (4)-(8) as objective functions in a mathematical optimization subject to a cost constraint such as Eq. (12). For example, one could minimize the output of potential environmental impact per mass of product given by Eq. (7) subject to keeping the total cost from increasing. This can be expressed as,

Minimize $\left.\hat{I}_{\text {out }}^{N P}=\hat{I}_{\text {out }}^{N P} \underline{\dot{M}}^{\text {(out) }}, \underline{x}_{1}^{N P}, \underline{x}_{2}^{N P}, \cdots, \underline{x}_{n}^{N P}, \underline{\dot{P}}\right)$

subject to Eq. (12) where $\underline{\dot{M}}^{\text {out }}$ is the vector of mass flowrates for all the output streams, $\underline{x}_{i}^{N P}$ is the vector of mass fractions for non-product component $i$ in all 
output streams, and $\underline{\dot{P}}$ is the vector of mass flowrates for all products. The optimization could involve the variation of operating variables and modification of the flowsheet configuration both. In this way one can systematically reduce the pollution indexes even in very complex processes. There are several robust algorithms such as simulated annealing (Kirkpatrick et al., 1983; van Laarhoven \& Aarts, 1987) that can be successfully used along with mixed integer programing (Grossmann, 1985, 1990) here.

\section{Conclusions}

A general theory and a methodology for incorporating pollution reduction into chemical process design has been presented. The work is still at an early stage of development particularly with respect to its application. However, the fundamental bases along which future work will proceed have been established.

When used in conjunction with chemical process simulators, the WAR algorithm offers a powerful methodology for evaluating the potential environmental impact of alternative process flow sheets. Although, the WAR algorithm is intended for use as part of a good faith effort to reduce the environmental foot print of process plants, and it does not obviate the need to make judicious engineering and environmental decisions. For example, there is no completely unambiguous way of setting values for the impact weighting factors or $\alpha_{l}$ 's. The reason is that the $\alpha_{l}$ 's represent the value that society places on particular types of environmental impacts, and this will vary across locations, cultures, and even time. One should point out, however, that engineering design practice has always used human judgement in determining any number of design parameters like safety factors. and, therefore, this dilemma is not new.

Nevertheless, there is a need to further improve the methodology for estimating potential environmental impacts and the weighting factors, there is a need to incorporate economics into the analysis, and there is a need to include computer aided process design and optimization. Future work will address these issues. The case studies, however, do illustrate that even in its present state the methodology is a useful process design tool.

\section{Acknowledgements}

The authors wish to acknowledge the support of Chemstations, Inc., Houston, Texas under a Cooperative Research and Development Agreement with the U.S. Environmental Protection Agency. The authors also thank Dr Douglas Young at the U.S. Environmental Protection Agency for critiquing the manuscript.

\section{Appendix A. Notation}

$C_{c} \quad$ Capital costs associated with a chemical process, monetary

$C_{o} \quad$ Operating costs associated with a chemical process, monetary

$I_{\text {syst }} \quad$ Potential environmental impact content of system, impact

$\dot{I}_{\text {in }}$

$\dot{I}_{\text {out }}$

$\dot{I}_{\text {gen }}$

$\dot{I}_{\text {in }}^{(i)}$

$\dot{I}_{\mathrm{gen}}^{N P}$

$\dot{I}_{\text {out }}^{N P}$

$\dot{I}_{\text {in }}^{N P}$

$\hat{I}_{\mathrm{gen}}^{N P}$

$\hat{I}_{\text {out }}^{N P}$

$\dot{M}_{j}^{(i)}$

$\underline{\dot{M}}^{\text {out }}$

$\hat{M}_{\text {gen }}^{N P}$

$\hat{M}_{\text {out }}^{N P}$

$\dot{P}_{p}$

$\underline{P}$

$x_{k j}$

$x_{k j}^{N P}$

$\underline{x}_{i}^{N P}$

$\alpha_{l}$

$\Phi_{n} \quad$ Pollution index, $\mathrm{kg} \mathrm{kg}^{-1}$

$\sigma_{j}$

$\psi_{j}$ Potential environmental impact input rate, impact $\mathrm{h}^{-1}$

Potential environmental impact output rate, impact $\mathrm{h}^{-1}$

Potential environmental impact generation rate, impact $\mathrm{h}^{-1}$

Potential environmental impact flow of stream $j$ input $(\mathrm{i}=i n)$ or output $(\mathrm{i}=$ out), impact $\mathrm{h}^{-1}$

Potential environmental impact generation rate by non-products, impact $\mathrm{h}^{-1}$

Potential environmental impact output rate due to non-products, impact $\mathrm{h}^{-1}$ Potential environmental impact input rate due to non-products, impact $\mathrm{h}^{-1}$ Specific potential environmental impact generation from non-products, impact $\mathrm{kg}^{-1}$

Specific output of potential environmental impact due to non-products, impact $\mathrm{kg}^{-1}$ Mass flow rate of stream $j$, input ( $i=$ in) or output ( $i=o u t), \mathrm{kg} \mathrm{h}^{-1}$ Vector of mass flowrates for all output streams, $\mathrm{kg} \mathrm{r} \mathrm{h}^{-1}$

Specific generation of non-product mass, $\mathrm{kg} \mathrm{kg}^{-1}$

Specific output of non-product mass, $\mathrm{kg} \mathrm{kg}^{-1}$

Mass output rate of product $p, \mathrm{~kg} \mathrm{~h}^{-1}$ Vector of mass flowrates for all products, $\mathrm{kg} \mathrm{h}^{-1}$

Mass fraction of chemical $k$ in stream j

Mass fraction of non-product $k$ in stream $j$

Vector of mass fractions for nonproduct component $i$ in all output streams, $\mathrm{kg} \mathrm{kg}^{-1}$

Chemical independent relative weighting factor for impact of type $l$, impact $\mathrm{kg}^{-1}$

Standard deviation of all chemical scores in impact category $j$, no units Overall potential environmental impact of chemical $j$, impact $\mathrm{kg}^{-1}$ 
$\psi_{j, l}^{s}$

Specific $(s)$ potential environmental impact of chemical $j$ for impact of type $l$

$(\text { Score })_{j, j}$

Relative potential impact score of chemical $i$ for impact of type $j$, no unit

$\left\langle(\text { Score })_{j}\right\rangle_{j} \quad$ Arithmetic average of the scores for all chemicals $i$ in impact category $j$, no units

\section{References}

Aspen Technology. (1997). Aspen Plus. Aspen Technology, Cambridge, Massachusetts.

Chemstations. (1997). Chemcad III, Ver. 3.2. Chemstations, Houston, Texas.

El-Halwagi, M. M., El-Halwagi, A. M., \& Manousiouthakis, V. (1992). Optimal design of dephenolization networks for petroleum-refinery wastes. Trans. I. Chem. E., 70b, 131.

Fonyo, Z., Kurum, S., \& Rippin, D. W. T. (1994). Process development for waste minimization: the retrofitting problem. Computers and Chemical Engineering, 18, S591.

Grossmann, I. E. (1985). Mixed-integer programming approach for the synthesis of integrated process flowsheets. Computers and Chemical Engineering, 9, 463.

Grossmann, I. E. (1990). Mixed-integer non-linear programming techniques for the synthesis of engineering systems. Research in Engineering Design, 1, 205.
Heijungs, R. (Final Ed.), Guinée, J. B., Huppes, G., Lankreijer, R. M., Udo de Haes, H. A. \& Wegener Sleeswijk, A. (1992). Environmental life cycle assessment of products guide October 1992. Centre of Environmental Science, Leiden.

Hilaly, A. K., \& Sikdar, S. K. (1994). Pollution balance: new methodology for minimizing waste production in manufacturing processes. Journal of the Air and Waste Management Association, 44, 1303.

ICARUS Corporation (1997). ICARUS Software. ICARUS Corporation, Rockville, Maryland.

Lapin, L. L. (1975). Statistics-Meaning and Method. HarcourtBrace-Jovanovich, New York.

Kirkpatrick, S., Gelatt, C., \& Vecchi, M. (1983). Optimization by simulated annealing. Science, 220, 670.

Lederman, P. B., \& Weaver, J. (1991). Pollution prevention through process modification: the challenge of the decade. AIChE Annual Meeting, LA, Paper 244a.

Mallick, S. K., Cabezas, H., Bare, J. C., \& Sikdar, S. K. (1996). A pollution reduction methodology for chemical process simulators. Industrial Engineering and Chemistry Research, 35, 4128.

Manousiouthakis, V., \& Allen, D. (1995). Process synthesis for waste minimization. AIChE Symposium Series, 304, 72.

Peters, M. S., Timmerhous, K. D. (1968). Plant design and economics for chemical engineers. McGraw-Hill, New York.

Richardson Engineering Services. (1997). Process Plant Construction Estimating Standards. Richardson Engineering Services, Mesa, Arizona.

Rossiter, A. P., 1995, Waste minimization through process design. McGraw-Hill, New York.

Van Laarhoven, P. J. M., \& Aarts, E. H. L. (1987). Simulated annealing: theory and applications. Holland: Reidel. 